\title{
Analysis of User Behavior on High-Resolution Tiled Displays
}

\author{
Robert Ball and Chris North \\ Center for Human-Computer Interaction, \\ Department of Computer Science, \\ Virginia Polytechnic Institute and State University, \\ Blacksburg, VA 24061 \\ \{rgb6, north\}@ivt. edu \\ http://infovis.cs.vt.edu
}

\begin{abstract}
The use of multiple monitors for personal desktop computing is becoming more prevalent as the price of display technology decreases. The use of two monitors for a single desktop has been shown to have performance improvement in several studies. However, few studies have been performed with more than three monitors. As a result, we report an observational analysis of the use of a large tiled display containing nine monitors (in a $3 \times 3$ matrix). The total resolution of the large display is $3840 \times 3072$, for a total of $11,796,480$ pixels. Over the course of six months we observed the behavior and actions of five users who used the display extensively as a desktop. We relate our observations, provide feedback concerning common usage of how people do and do not use the display, provide common scenarios and results of interviews, and give a series of design recommendations and guidelines for future designers of applications for high-resolution, tiled displays.
\end{abstract}

\section{Introduction}

Historically, large high-resolution tiled displays have been reserved for control rooms and large government facilities. However, using multiple monitors for one computer is becoming more common in both business and home life as monitors become less expensive and more research is done on productivity gains. With a small amount of extra effort and cost, a single computer can operate several monitors. For example, Microsoft Windows easily supports up to 10 monitors on a single machine.

Tiled displays, with greater numbers of pixels, have the potential to increase the quantity and granularity of displayed information. They allow more information, more applications, and high-resolution images and visualizations to be viewed. Along with additional screen space, they pose problems of being physically larger, more difficult to navigate, and often have bezels between tiled monitors that distort the image (see figure 1). A bezel is the plastic covering around the edges of the monitors.

Our motivation for this paper is to assess how large, high-resolution, tiled displays help individuals or small groups of users with everyday tasks, such as word processing, programming, and viewing images. Our goal is to gather initial basic evidence about how users adapt to the increased screen space and larger physical size of the displays, how they utilize the space and strategically organize their work, how they deal with the bezels between tiles, and the benefits and difficulties they have. 


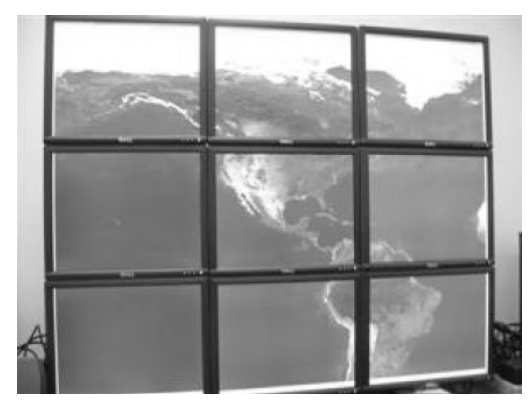

Fig. 1. Picture of the tiled display used in this analysis

In our analysis, a number of people extensively used a tiled display to view highresolution images, GIS data, and perform rudimentary tasks such as using word processors, email clients, and spreadsheets over the course of six months.

This paper differs from other studies on large displays in two ways. First, this paper looks at the use of large, high-resolution displays, not just physically larger displays such as projectors. Second, this paper evaluates the use of such a display for a long period of time, not just for 30 minute to 1 hour segments. In this paper we explain previous work, our analysis method, key observations, and a list of suggestions for user interface designers.

\section{Previous Work}

A variety of studies have been performed on large screens and multiple screens to compare their effectiveness to that of small or single screens. Figure 2 visually shows the different categories of research on different types of displays. In general, there are two independent variables that researchers look at: physical size of the display, and resolution (total number of pixels, not pixel density) of the display.

\subsection{Standard Projectors and Monitors}

Several studies have suggested that the increase of the physical size of a screen helps with memory. Lin, et al. [11] suggests that an increase of one's field of view increases one's sense of presence and memory. Raja, et al. [12] suggests benefits of being surrounded by large low-resolution screens (CAVE) when dealing with data visualization (information visualization).

Tan et al. show how performance on a large low-resolution screen can be better than a conventional small screen even at the same resolution. They show that with the same visual angle participants in a study were able to perform better on a large screen compared to a single monitor for both spatial performance [17] and 3D virtual navigation [16]. However, large, low-resolution screens have the problem that they can only show the same amount of data as small screens because they have similar resolutions. As a result, the data on the screen is simply enlarged. 


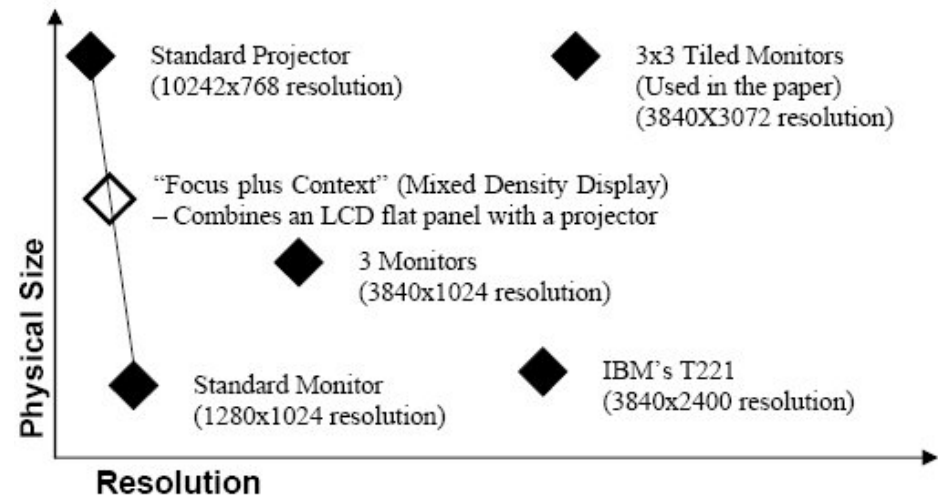

Fig. 2. The configuration space of displays, showing how several instances relate to each other with respect to physical size and total resolution (number of pixels)

Terri Simmons [14] conducted a study comparing performance on different-sized monitors (17 inch to 21 inch), with slightly differing resolutions. His results were unsurprising in that they suggest that people perform faster with the largest monitor, and slightly higher resolution, as opposed to the smaller monitors.

\subsection{Two to Three Monitors}

Czerwinski et al. [4] explain the current state of performance measurements and explain that their own study showed conclusively that participants using a multimonitor configuration affording increased resolution ( 3 monitors wide) performed better than on a single monitor. Tan, et al. [18] also show how retention can be increased by using extra screen space to display different images in the user's periphery to help recall more from a particular task session.

Some studies have also shown that gender can have an effect with spatial performance. Some studies by Czerwinksi, Tan, and Robertson show that the effects of an increase of field of view can offset gender bias [5]. Their findings indicate that women need a wider field of view than men to achieve the same performance.

\subsection{Mixed Density Displays}

In a unique study, Baudisch et al. [3] performed an experiment using their "Focus plus Context" screen to study the effects of having a small LCD screen embedded within a large projection screen (both standard low-resolution). In effect, they created a focus+context visualization using pixel density distortion instead of spatial distortion.

\subsection{Large Tiled Displays}

Few studies have reported findings on large tiled displays. Guimbretière, et al. [8] describe new interactive techniques for direct pen-based interaction on high- 
resolution displays, and reviews several other interaction techniques. A preliminary study on basic perceptual and navigational techniques shows that for fine-detailed information it is faster to find and compare information on high-resolution displays than low-resolution displays [1]. Our behavioral analysis falls into this category. Since little is known about how people use large high-resolution tiled displays, we chose to observe a variety of people using it for common tasks in a longitudinal study.

\section{Analysis Method}

In order to analyze everyday use of physically larger, high-resolution displays, we first constructed a 3x3 tiled display of LCD flat panels approximately 37 inches (94 $\mathrm{cm})$ tall and 44.4 inches $(113 \mathrm{~cm})$ wide. We put the display on a standard desktop table approximately 30 inches $(76 \mathrm{~cm})$ off the ground. We then allowed a variety of people to use it, and observed its use over a six month period.

With modern plug and play technology, creating a large high-resolution tiled display takes relatively little expertise and is fairly low cost. Modern operating systems such as Windows and Linux have built-in support for multiple monitors.

Our tiled display was constructed from nine 17" Dell monitors affixed to a wooden frame (see figure 3). We used one computer, a Dell Optiplex GX270, to support all nine monitors. In addition to the dual head AGP video card that came with the computer we installed four additional PCI video cards. All video cards were NVidia GeForce FX 5200.

Figure 3 show various aspects of the tiled display. The picture on the left shows a side angle of the monitors as mounted on a wood frame. The same display could also be mounted on a supported wall or other more permanent place. However, we desired to have a display that could be easily relocated or adjusted. The picture on the right shows a front view of the tiled display with one of the monitors removed to show the underlying frame.

Five participants used the nine-monitor tiled display for at least three months in a form of time sharing. These users will be referred to hereafter as power users. One power user was designated time to use the tiled display during morning and early afternoon and used it as his primary workspace while the other four participants routinely reserved time during all other hours of the day and night on a first come, first serve basis as a secondary workspace. Each of these participants were researchers that used the tiled display for a variety of reasons from normal daily activities such as reading/writing email, browsing the Internet, writing papers, programming, viewing images, and running experiments. Each of these participants was a graduate student conducting research in computer science. In addition, there were four undergraduate users that used it for collaboration work in setting up experiments that also used the display on a first come first serve basis after the first three months of the analysis. 

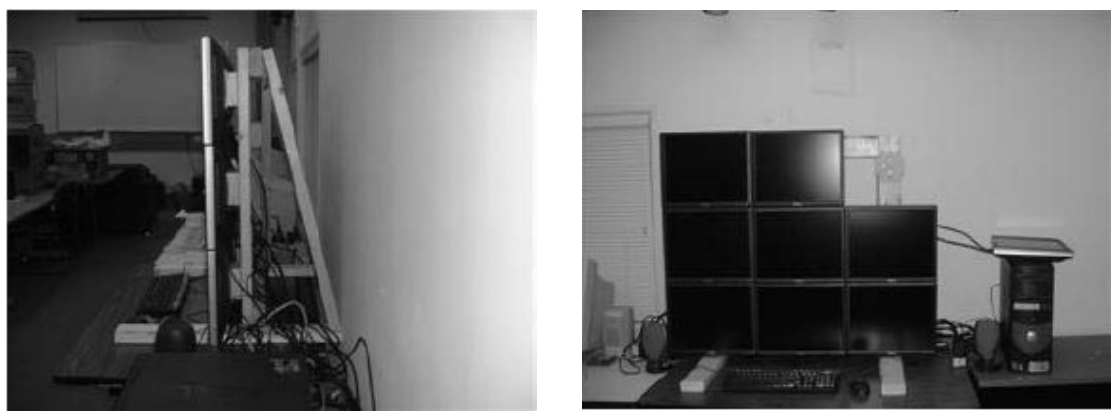

Fig. 3. The left figure shows the side view of the tiled display, showing how the monitors connect to the frame. The right figure shows the front view of the tiled display, with one monitor disconnected to show the underlying wooden frame. The sole computer powering the display is shown to the right.

During the six month period we performed three experiments on how well individuals performed and interacted on the tiled display at different configurations using one, four, and nine monitors. Although these experiments were not done for the purpose of this paper, we were able to take our observations from the different experiments and apply them here. There were approximately 65 individuals that participated in the three experiments. Each of these individuals spent between 30 minutes to an hour interacting with the tiled display. Our analysis also draws from our experiences with dozens of other people that were given demonstrations. Such people included high school students, people from the community, government officials, business professionals, and university students, faculty and staff.

Our observations in this paper are a result of a broad range of observations with the above mentioned people, ranging in form from the following methods:

- Direct observations as individuals interact with the tiled display.

- Formal interviews involving question and answer.

- Informal interviews to let the user talk about their experience without asking leading questions.

\section{Observations}

This section enumerates key observations during the course of our analysis. In this section we only highlight common benefits and disadvantages.

\subsection{Performance}

From the previous work section, many researchers have shown the advantages of having larger displays. Several researchers have shown that larger displays improve performance with different tasks including multi-tasking [4], spatial orientation [17], and general usability [14][7] to name a few.

Our observations show that users clearly referenced back and forth between primary tasks and secondary tasks on a regular basis. Also, interviews show that 
people reported having to do less window management and were more satisfied with the display as a result.

Our experience concurs with [7], showing that having a larger high-resolution desktop decreases the time switching between applications, allowing for a decrease in cognitive load. When a user is performing a specific task they often have the need for supporting tasks. For example, when writing a document, support information (e.g. references) is often needed. By having extra screen space, a user is capable of simply glancing at the supporting information instead of the need of switching between applications. By not performing extra virtual navigation, less concentration is lost.

Another example of decreased cognitive load is viewing large images and visualizations. Many large images are too large for the entire image to be shown at once on one or two monitors, requiring additional navigation to see the entire image. With a large display there often is no need to navigate the image as the entire image can be seen all at once.

\subsection{More Viewable Information at Once}

With an increase in screen space comes many different opportunities for increased spatial positioning. With one monitor, users can only have a few applications viewable at a time. In contrast, with multiple monitors there arises an opportunity to not only have more applications viewable, but have each of those applications taking up more screen space than is available to one monitor.

Our actual observations show that users often take advantage of the increased screen space. An observed scenario that represents common usage among most users follows: A user logs into the machine running the large display. He then opens up his remote desktop (for the purpose of using his own email client) and positions it in the lower-right monitor as he always does. He then opens a word processor to continue writing a report. Over the course of writing his report he also opens three Internet browsers, and a spread sheet. All the different opened applications, including the remote desktop, support his main task of writing the report. The different applications each occupy a monitor with the remote desktop always remaining in the lower-right monitor and his word processor always being in the middle monitor.

This actual scenario taken from a session of a user that uses the display as a secondary workplace accurately represents most users. Key results from observations and interviews show that users prefer to use the middle monitor for their main application, surround the main application with support applications, and maintain constant positioning for certain applications (e.g. email, instant messengers, etc.).
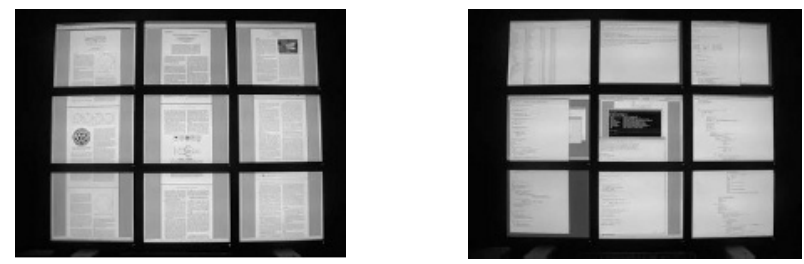

Fig. 4. The picture on the left shows three documents being displayed at once for easy comparison. The picture on the right shows a user's programming environment. 
Two examples of using the increased screen space can be seen in figure 4 . The picture on the left shows three PDF applications open side by side. Three different conference papers are viewed simultaneously allowing for easy comparisons of the documents. The picture on the right is a picture of actual usage while programming. Several applications can be seen including a command prompt for compiling purposes in the center, several programming editors, and one file explorer.

\subsection{Categorization of Regions}

After time, users tend to dedicate certain regions of the screen for certain applications. For example, one power user always positioned his email client application on the middle-right monitor while another power user always positioned his remote desktop (to get to his email client) on the lower-right monitor. After a period of time, users tend to develop preferences of where certain applications should be located. This preference of positioning applications in the same place relies on people's spatial memory abilities. This use of spatial memory is similar to the concept explored in Robertson's Data Mountain [13].

This use of positioning of applications was also observed to follow categories. In other words, power users tended to keep many personal or nonessential tasks in the same regions of the screen. Email, calendars, and instant messengers tended to have positions that were further away from the center of the screen and more toward the periphery.

\subsection{Focus on Center}

The primary task at any given time with the tiled display tended to be in the center column of the tiled display. It was observed that if a secondary application that was initially further away from the center column became more important and commanded more attention then it tended to be moved closer to the center column.

\subsection{Bezel Adaptations}

Bezels, the plastic border separating monitors, are often considered a distraction to users. Current LCD technology makes it very difficult to construct a large tiled display without bezels (figure 5). However, interviews from users and observations appear to differ as to how much a distraction they are. Key results from observations and interviews show that users do not generally like bezels, but that they use them very efficiently to their advantage to align, segregate, and differentiate applications.

With a single monitor the bezel does not generally cause any concern and usually goes unnoticed. On the other hand, the bezels around tiled monitors are usually one of the first things that people notice. The thickness of the bezels between monitors is a limiting factor on how close monitors can be tiled.

Interviews from users indicate that bezels are an inconvenience, irritation, and a point of frustration. Users have pointed out that bezels can distort the size of a document or an image. This distortion gives documents or images an artificial lengthening and can be confusing. As one user explained, "It can be really tough to mentally block the bezels out of your mind." 
However, our observations show that bezels tend to help users quickly segregate applications between monitors. A common scenario for all users, whether first time users or power users of the display follows: Users first open the primary application in the center monitor. Users then either maximize the application to fill the center monitor with the maximize button or drag the application by hand to approximately fill the center monitor. All subsequent applications that are opened are usually maximized in a monitor that is not in use.
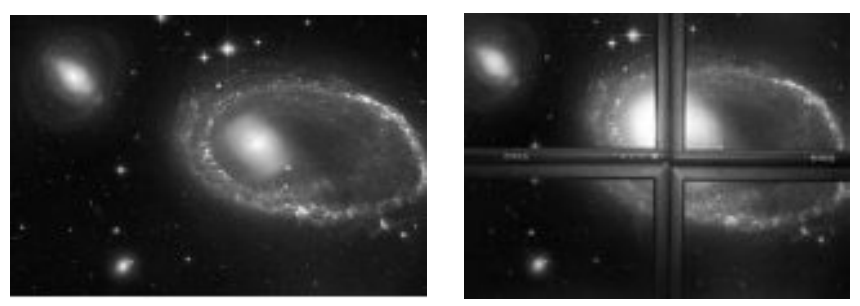

Fig. 5. Comparison of an image with and without bezels splitting up the image

Only the most experienced user, the power user who used the display as a primary workspace, consistently used applications across bezel boundaries. The primary power user gradually became more comfortable crossing bezel boundaries and was able to grow accustomed to text and images being separated by bezels over a course of months. In contrast, our observations and interviews show that most users were able to use bezels to separate applications from the first time they used the display.

\subsection{Adjustment Period - From Small to Large}

Key difficulties that new users struggle with are moving beyond the paradigm of one monitor, finding the cursor, using space efficiently, and other unpredicted behavior from diverse applications.

One example scenario that took place is when a student showing a demo on the tiled display had two applications open. One application was a control panel driving the other application, which showed a visualization of data. The student had nine monitors of screen space, but only utilized one of them. The student had the two applications maximized to fit on the center monitor. He constantly brought the control panel to the front (over the visualization application), modified one or two parameters and then brought the visualization back to the front. As the student had eight other monitors to use, he could have easily used another monitor to show and interact with the control panel without the need to block the visualization window. When asked about it, the student responded with surprise and simply had not considered the possibility of spreading out the application over multiple tiles.

Adjusting to the larger display takes a variable amount of time. For example, moving beyond the paradigm of using only one monitor usually took less than an hour while finding the best strategies to finding the cursor usually took weeks to learn.

As learning how to use the display takes time and is not instantaneous, a new user may get discouraged by actual loss of productivity at first. As a few applications no 
longer appear to act as they did, or do not act as the new user would think, the experience can be frustrating. Unlike the noted publications that claim performance boosts with larger displays, new users are not necessarily given a lengthy tutorial at the onset for each application they might use, as was the case in the publications.

\subsection{Adjustment Period - From Large to Small}

Although not intuitive, we have also noted a definite frustration when using smallersized displays after using the larger display for an extended period of time. One scenario from the primary workspace follows: After approximately four months of using only the tiled display as his desktop he encountered a time when he had to prepare for a presentation on a laptop. When trying to change a small program and write notes about the changes, he encountered a great deal of frustration when not being able to see both the program he was editing and the word processor he was using for taking notes at the same time. It was also necessary at the time to have several more applications open at the same time on the laptop and he found himself frustrated and confused as to how to best manage the different applications.

When using only one monitor one power user reported "feeling cramped." Another power user said, "[I would] get really close to the laptop to make it bigger." Users had unlearned their previous organization strategies and had difficulty relearning them.

\subsection{Collaboration Usage}

Through the increase in actual screen size, more people can gather around the display at once. However, although the display affords many uses for collaboration, we observed only the following collaborations in the order of most often to least often: image viewing, visualization viewing, Internet browsing, and document writing.

A normal scenario of collaboration usage is two people working on a document. One person drives the display while the other person is on the right of the first person. Most of the applications are found in four monitors to the bottom and right as any applications found on the left of the person driving the display are hard to see for the person on the right. These users found it helpful to be able to review long documents together by stretching the windows vertically across multiple monitors and pointing with their hands to portions of the text.

\subsection{Maximizing an Application}

There are several assumptions that operating system and application writers have made that do not hold true for displays of more than one monitor. Many different assumptions about having only one monitor have been made that appear anti-intuitive with several monitors.

For example, a scenario that occurred with almost every user follows: When a user tries to maximize an application across all nine monitors, they usually click on the "maximize" button in the top right-hand corner of the application. Most users expect to have the application maximize to the full screen size. However, what occurs is that the application maximizes to fill up a single monitor. 


\subsection{Dialogue Boxes}

A problem with applications is their dialogue boxes. Dialogue, or pop-up boxes, may appear any where on the screen space and may be difficult to find. Using only one monitor it is often trivial to find a dialogue box as there is very little area for it to appear. However, with multiple monitors, such as the tiled nine that we used, it can be difficult to find or even notice that a dialogue box was opened if not expecting it. It has been the experience of some users to feel frustrated when suddenly it appears that their application is no longer responsive, when in fact there exists a modal dialogue box some where in the screen space that requires attention.

\subsection{Navigational Issues}

Interviews with users, including power users and new users, indicate that losing one's mouse cursor on the screen is common and frustrating. To find the mouse cursor on the screen, users would perform one of the following common strategies, listed from most often used to least often used: Quickly moving the mouse back and forth in a small area to use human visual motion detection, simply scanning the entire display until finding it, and moving the mouse to an extreme edge (e.g. moving the mouse down and right until finding it in the bottom right corner of the screen).

Using even two monitors at a time can lead to confusion when using a mouse. Simply put, users can easily lose track of where the cursor is on the screen. For nine monitors the problem is further exacerbated. Several ideas have risen to solve this problem, such as increasing the visibility of the mouse cursor at all times [3].

Windows XP has a feature that helps the user to detect the mouse when it is lost. By pressing and releasing the "CTRL" key without any other key combination, an animated circle grows and shrinks around the cursor. However, our experience shows that such a feature is practically useless on a large tiled screen because the user has to be looking at the correct display tile to detect the animation. For example, if a user is looking for the cursor at the top right of the display and the cursor is in the bottom right then the animated circle cannot be seen by peripheral vision.

However, in general, less navigation is required in large high-resolution displays. By having a larger display, the needs to navigate at all are decreased. For example, as mentioned before, looking at a large high-resolution image, the entire image, or a greater percent of the image can be shown at once, decreasing the need to navigate as much as on a smaller display. The same is also true for documents, web browsing, etc.

\subsection{Physical Size and Layout}

A disadvantage of the high-resolution tiled display is its cumbersome physical size. Common questions such as where one should put the display are nontrivial. Often office space is considered sacred and not easily granted at many businesses and universities. Although most tiled displays are tiled LCD's (liquid crystal displays), and thus take up less room than CRT's (cathode ray tubes), most tiled displays would not fit in office cubicles.

Also, there is a potential for additional physical stress and pain. If using a keyboard or a mouse for extended periods of time cause problems, then it is also logical that extended use with a large display may cause physical injury or discomfort to the neck 
or back although we have not observed any problems yet. More research is needed in this area before concluding anything about physical stress caused by large displays.

\section{Suggestions and Recommendations}

Through the course of our analysis we have learned many things about the use of large high-resolution displays. In this section we discuss several suggestions and recommendations as guidance to future designers.

\subsection{Number of Monitors and Physical Layout}

Although biased, we concur with [7 that people should use two monitors as a minimum for each desktop. As a maximum, the layout and size of the monitors come into play. For example, experimenting with different layouts we found that six monitors side by side, is very difficult to use. However, the same 6 monitors can be used very efficiently when stacked on top of each other in a $2 \times 3$ array.

Several papers including [7] show performance increases with as few as two or three monitors. Also, Simmons [14] shows that just modestly increasing the resolution helps. [17] shows that just increasing the physical size is better for spatial performance. In general, abundant literature exists to show that modern desktop displays are inadequate for maximum performance.

As we have shown previously, we believe that unless a user has needs to view large images on a constant basis, no more than six monitors is recommended. This recommendation is based on physical size of the monitors and not on resolution density. However, with curved displays the number of useful monitors may increase. Our preliminary studies show that having a curved display helps people use more of the outer regions of the display.

\subsection{Notification Systems and Controls}

Small notifications are not seen on a large, high-resolution display. As a result, we suggest that notifications should be redesigned for large, high-resolution displays. They should be larger, and grouped together in a central area instead of being spread out. For example, an application that has notification systems and controls that are located on the edges of the application might be acceptable for one monitor; however, for large high-resolution displays the controls or notification systems might be several feet from each other. Also, dialog boxes (e.g. popup windows) should be presented in a more uniform manner [10].

\subsection{Use of Bezels}

Bezels are the plastic surrounding the outside of monitors. Bezels provide natural separations between applications and tasks and can be regarded as helpful. Currently operating systems hide all knowledge of more than one monitor from applications. However, if applications were aware of where the monitor separations are they could take advantage of that information and display things more intelligently. 


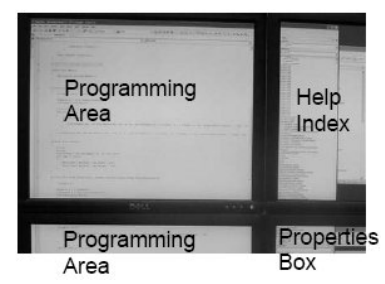

Fig. 6. An example of using bezels to separate work spaces in the Microsoft Visual Studio .NET programming environment

Users can also take advantage of bezels by segregating their work. As explained earlier, users reported using different regions of the screen to separate tasks and ideas. This concept can be taken farther by using bezels to also separate parts of the same application. As briefly mentioned by [7], movable taskbars can increase the amount of available screen space. Also, even with taskbars are not moveable, bezels can be used in such as way to increase work areas as can be seen in figure 6 . Internal subwindows can be maximized to an entire tile, pushing toolbars and supporting frames across the bezel to other tiles.

\subsection{Mouse}

It is clear that the current state of interaction with computers, with only the mouse as input, is inadequate. Interviewing users of another large tiled display (with 40 tiled displays) from another organization confirms the need. For example, scrolling the mouse across 10 feet of high-resolution screen space is absurd.

Finding the mouse can be problematic. One suggestion is to increase the size of the mouse or the animation finder to be more helpful. In Microsoft Windows' "CTRL" key solution, the animation circle size should be more noticeable and larger as the screen size is larger. It should also be customizable for different sized displays.

For the time being, our recommendation to users for quickly locating one's mouse is to scroll to a corner. For example, if the user chose the bottom right corner of the screen then the user would rapidly scroll down and right. After about two quick scroll movements, the cursor can be found at the desired corner.

Using other interactive techniques, such as laser pointers, pen-based interactions, touch sensitive panels, etc. may be more appropriate than using a mouse for large displays. Unfortunately, these other techniques are either found only in research environments or are prohibitively expensive for common use.

\section{Conclusion}

Our behavioral analysis shows that a large high-resolution display affords a number of advantages. This section summarizes the broad range of advantages and disadvantages explained in this paper. Advantages include: Improved user performance for task switching or viewing large documents, increased ability to spatially position applications and shortcuts for quick access and recall, bezel adaptations for easy separation of tasks, increased ability to work collaboratively, 
increased screen space for awareness for secondary tasks (e.g. Email, instant messengers, news, etc.), and enjoyment - interviewed users almost unanimously prefer multiple monitors to one.

Disadvantages include: Adjustment periods (both from one monitor to multiple monitors, and vice versa), more screen space wasted, unpredicted behaviors with software applications including notification systems and dialog boxes, navigational issues with losing the mouse, input focus, or other highlights, and physical size and layout may require more physical strain

\section{Acknowledgements}

This research is partially supported by the National Science Foundation grant \#CNS04-23611. This study was also supported and monitored by the Advanced Research and Development Activity (ARDA) and the National Geospatial-Intelligence Agency (NGA) under Contract Number HM1582-05-1-2001. The views, opinions, and findings contained in this report are those of the authors and should not be construed as an official US Department of Defense position, policy, or decision, unless so designated by other official documentation.

\section{References}

1. Ball, Robert and North, Chris. "Effects of Tiled High-Resolution Display on Basic Visualization and Navigation Tasks." In Extended Abstracts CHI'05, p. 1196-1199.

2. Baudisch, P. Good, N., Bellotti, V., Schraedley, P. "Keeping things in context: a comparative evaluation of focus plus context screens, overviews, and zooming." In Proc. of CHI '02. p. $259-266$.

3. Baudisch, P. Cutrell, E., Robertson, G. "High-Density Cursor: A Visualization Technique That Helps Users Keep Track of Fast-Moving Mouse Cursors." Proc. of INTERACT '03. p. 236-243.

4. Czerwinski, M., Smith, G., Regan, T., Meyers, B., Robertson, G., Starkweather, G. "Toward characterizing the productivity benefits of very large displays." In Proc. of Interact'03.

5. Czerwinski, M. Tan, D., Robertson, G. "Women take a wider view." In Proc. of CHI 'O2, p. $195-201$.

6. Furnas, G., Bederson, B. "Space-scale diagrams: Understanding multiscale interfaces." In Proc. of CHI '95 Human Factors in Computing Systems, p. 234 - 241.

7. Grudin, J. Partitioning Digital Worlds: Focal and Peripheral Awareness in Multiple Monitor Use. Proc. CHI 2000. p. 458-465.

8. Guimbretière, F. Stone, M. and Winograd, T. "Fluid Interaction with High-resolution Wall-size Displays." In Proceedings of UIST 2001, 21-30, ACM Press.

9. Healey, C., Booth, K., Enns, J. "High-speed visual estimation using preattentive processing." ACM TOCHI, volume 3 (2), June 1996, p. 107 - 135.

10. 10. Hutchings, D., Stasko, J. "mudibo: Multiple Dialog Boxes for Multiple Monitors. ” In Extended Abstracts of CHI'05. p. 1471-1474.

11. Lin, J., Duh, H., Parker, D., Abi-Rached, H., Furness, T. "Effects of view on presence enjoyment, memory, and simulator sickness in a virtual environment." In Proc. of IEEE Virtual Reality 2002. 
12. Raja, D., Bowman, D., Lucas, J., North, C. "Exploring the benefits of immersion in abstract information visualization." In Proc. Of IPT (Immersive Projection Technology), 2004.

13. Robertson, G., Czerwinski, M, Larson, K., Robbins, D., Thiel, D., van Dantzich, M. "Data Mountain: Using Spatial Memory for Document Management." Proc. of UIST '98. p. 153162.

14. Simmons, T. "What's the optimum computer display size?" Ergonomics in Design Vol. Fall 2001, p. $19-25$.

15. Tan, D., Czerwinski, M. Robertson, G. "Women go with the (optical) flow." In Proc. of CHI '03, p. $209-215$.

16. Tan, D., Gergle, D., Scupelli, P., Pausch, R. "Physically large displays improve path integration in 3D virtual navigation tasks." In Proc. of CHI '04. p. 439 - 446.

17. Tan, D., Gergle, D., Scupelli, P., Pausch, R. "With similar visual angles, larger display improve spatial performance.” In Proc. of CHI '03. p. 217 - 224.

18. Tan, D., Stefanucci, J., Proffitt, D., Pausch, R. "The infocockpit: providing location and place to aid human memory." In Proc. of PUI 2001. p. 1 - 4. 